\title{
An ultrafast carbon nanotube terahertz polarisation modulator
}

\author{
Callum J. Docherty, ${ }^{1}$ Samuel D. Stranks, ${ }^{1}$ Severin N. Habisreutinger, ${ }^{1}$ Hannah \\ J. Joyce, ${ }^{1}$ Laura M. Herz, ${ }^{1}$ Robin J. Nicholas, ${ }^{1}$ and Michael B. Johnston ${ }^{1, *}$ \\ ${ }^{1}$ Department of Physics, University of Oxford, \\ Clarendon Laboratory, Parks Road, Oxford OX1 3PU, U.K.
}

\begin{abstract}
We demonstrate ultrafast modulation of terahertz radiation by unaligned optically-pumped single-walled carbon nanotubes. Photoexcitation by an ultrafast optical pump pulse induces transient terahertz absorption in nanowires aligned parallel to the optical pump. By controlling the polarisation of the optical pump, we show that terahertz polarisation and modulation can be tuned, allowing sub-picosecond modulation of terahertz radiation. Such speeds suggest potential for semiconductor nanowire devices in terahertz communication technologies.
\end{abstract}

${ }^{*}$ Electronic address: m.johnston@physics.ox.ac.uk 
Whereas microwaves and near-infrared light have been widely used in communication technologies, the terahertz region of the electromagnetic spectrum, lying between microwaves and IR, has been notably underused. Historically, intense sources of $\mathrm{THz}$ waves, with a frequency from $50 \mathrm{GHz}$ to $10 \mathrm{THz}$, have been difficult to find [1,2]. A range of sources, from optical rectification to quantum cascade lasers are now available $[1,3]$, but high speed $\mathrm{THz}$ communications have yet to be realised. A lack of satisfactory practical THz modulators, required to encode information in the $\mathrm{THz}$ wave, is a chief reason for the lack of progress $[4,5]$.

Terahertz intensity modulators have already been demonstrated using a range of approaches [5, 6], including optically pumped silicon wafers [7-9] and electrically modulated metamaterials $[10,11]$. Liquid crystals have been suggested for use as terahertz polarisation modulators $[12,13]$. However these methods do not provide fast enough switching speeds to exploit the full potential of $\mathrm{THz}$ communication applications, where picosecond scale switching is desirable. On the other hand, static THz polarisers have also been developed, for example by arranging fine wires in a grid $[14,15]$, but again cannot provide fast switching speeds.

Carbon nanotubes may offer a solution to the problem of fast switching. It has been shown that highly aligned single-walled carbon nanotubes (SWNTs) can act as optical [16, 17] and $\mathrm{THz}$ polarisers $[18,19]$, utilising the intrinsic anisotropy of these one-dimensional materials. Previously, we have shown that photoexcitation of highly aligned semiconducting nanotubes can provide dynamic THz polarisation [20]. Photoexcitation of these nanotubes creates an exciton along the length of the nanotube, which can absorb $\mathrm{THz}$ radiation aligned with it during the lifetime of the exciton (10-100 ps [21-23]). Rotation of such a nanotube structure allows selective transient polarisation of incident $\mathrm{THz}$ waves.

However, our previously reported device required the nanotubes to be highly aligned, and only allowed modulation of $\mathrm{THz}$ intensity by rotation of the nanotube grid. Here, we present ultrafast dynamic $\mathrm{THz}$ modulation by an unaligned nanotube device. Selective photoexcitation of the nanotubes by a polarisation-controlled optical pump creates a transient and tunable $\mathrm{THz}$ polariser which can modulate $\mathrm{THz}$ waves on a sub-picosecond timescale.

The SWNT sample was produced using a fast, simple and cheap method described previ- 

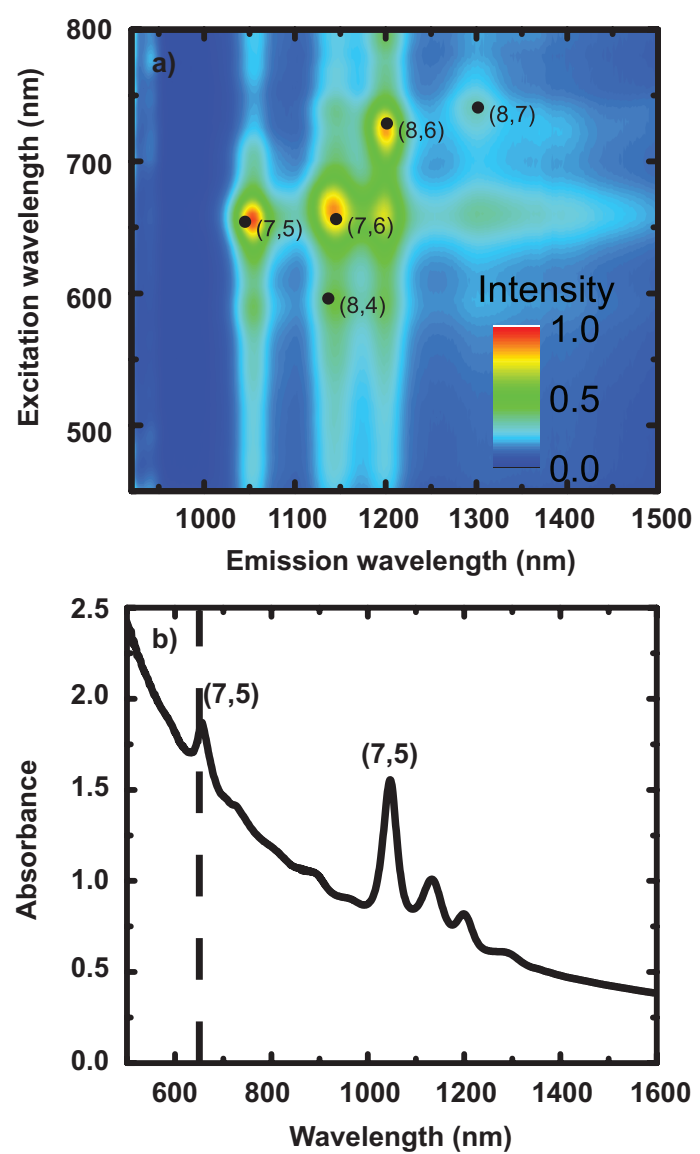

FIG. 1: Characterisation of the SWNT dispersion. a) Photoluminescence emission spectroscopy of the nanotube sample. A very narrow distribution of semiconducting chiralities is apparent. b) Absorption spectrum of the nanotubes. Photoexcitation by the $650 \mathrm{~nm}$ pump used in the opticalpump $\mathrm{THz}$-probe measurements (represented by the dashed line) selectively excites only the $(7,5)$ nanotubes.

ously [24-26]. Briefly, a narrow chirality distribution of semiconducting single-walled carbon nanotubes was produced by selectively dispersing the nanotubes with the polymer poly $(9,9$ dioctylfluorenyl-2,7-diyl) (PFO). The polymer and SWNT mixture was agitated using an ultrasonic probe, leading to a selective wrapping of semiconducting species. Unwrapped and bundled SWNTs precipitate out of solution and were removed via centrifugation. High yields of polymer-wrapped SWNTs were obtained by optimizing the polymer:nanotube ratio and the sonication time. The polymer-nanotube blends were then simply drop-cast from the o-xylene dispersions on to z-cut quartz discs while gently heating the substrates at $60^{\circ} \mathrm{C}$ to create an unaligned SWNT sample. Z-cut quartz was selected for its high transmission of 
$\mathrm{THz}$ radiation, and unresponsiveness to photoexcitation.

Figure 1 shows the characterisation of this SWNT film. Photoluminescence emission (PLE) spectroscopy was used to determine the chirality of nanotubes present in the sample. As corroborated by the absorption spectrum in Figure 1b, photoexcitation by a $650 \mathrm{~nm}$ pump, as used in the $\mathrm{THz}$ measurements, selectively photoexcites the $(7,5)$ semiconducting nanotubes.

The transient $\mathrm{THz}$ modulation provided by the SWNT sample was determined using a time domain $\mathrm{THz}$ spectrometer described previously [27]. THz radiation was generated by optical rectification in a $2 \mathrm{~mm}$ thick GaP crystal, photoexcited by $800 \mathrm{~nm}$ femtosecond pulses [1]. The electric field of the $\mathrm{THz}$ radiation was detected by electro-optic sampling in a second GaP crystal, and the difference in $\mathrm{THz}$ transmission through the photoexcited SWNT sample and the sample without photoexcitation determined by lock-in detection referenced to a chopper in the pump beam. The $650 \mathrm{~nm}$ pump pulses used to photoexcite the nanotube modulator were generated with a fluence of $175 \mu \mathrm{J} / \mathrm{cm}^{2}$ by a TOPAS optical parametric amplifier, pumped from an $800 \mathrm{~nm}$ Ti:Sapphire regenerative amplifier.

The nanotube sample was photoexcited by femtosecond pulses of the $650 \mathrm{~nm}$ polarised pump beam. Due to the inherent anisotropy of the nanotubes, nanotubes aligned in the same direction as the pump pulse polarisation are preferentially photoexcited. The resulting exciton population resides predominantly on nanotubes aligned in the direction of the pump polarisation, and hence incident $\mathrm{THz}$ radiation also polarised in this direction will be preferentially absorbed by these carriers. Thus, by selecting the polarisation of the pump beam, we were able to control the $\mathrm{THz}$ modulation capability of the nanotube sample.

The polarisation of the pump beam was controlled by a half waveplate placed in the beam before the nanotube sample. Rotating the waveplate by an angle $\theta$ rotated the pump beam polarisation by an angle $2 \theta$ - i.e. the pump beam was rotated from vertical polarisation to horizontal polarisation by rotating the waveplate by $45^{\circ}$. This measurement is schematically demonstrated in Figure 2b.

In Figure 2c, we present the effect of rotating the waveplate on the $\mathrm{THz}$ modulation through the nanotube sample. The ratio of change in $\mathrm{THz}$ transmission intensity due to photoexcitation to the transmission through the non-photoexcited sample, $\Delta T / T_{\mathrm{OFF}}$, is plotted as a function of waveplate angle. Here, $\Delta T=T_{\mathrm{ON}}-T_{\mathrm{OFF}}$, where $T_{\mathrm{ON}}$ and $T_{\mathrm{OFF}}$ represent the $\mathrm{THz}$ transmission of the sample with and without photoexcitation respectively. 

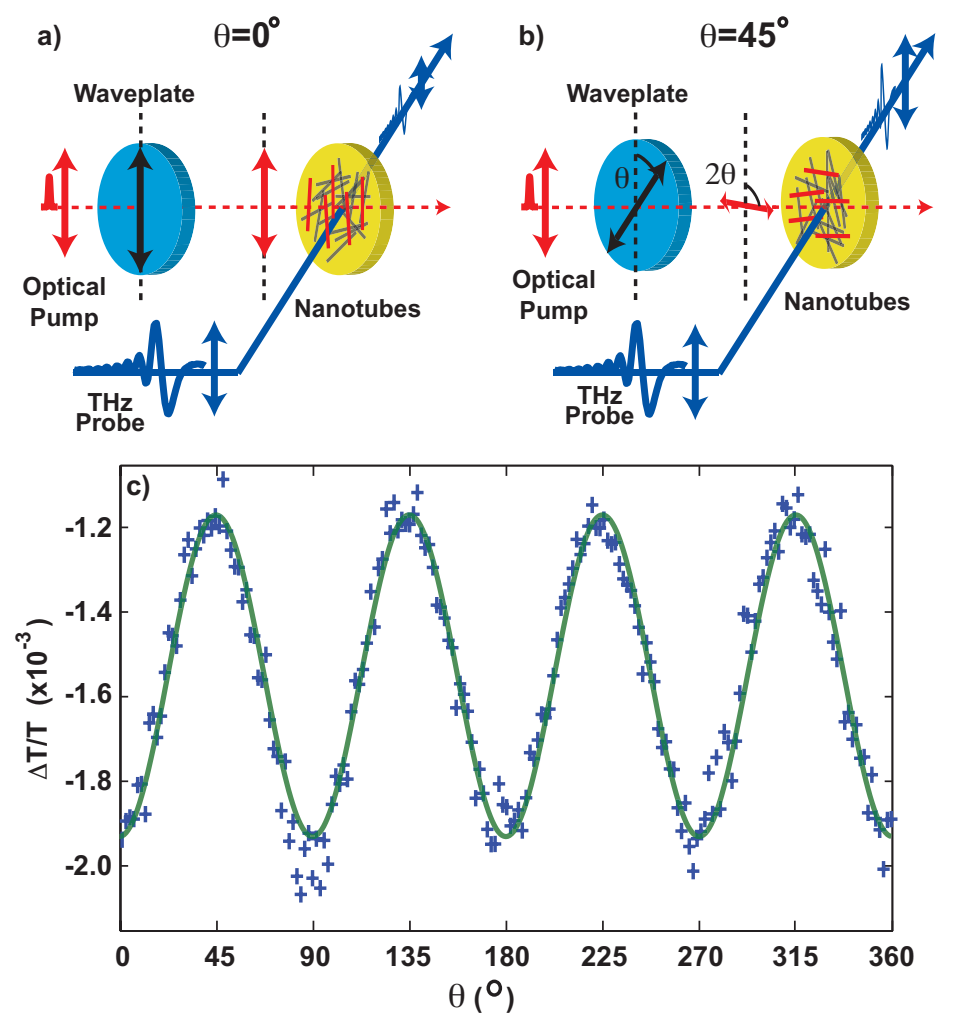

FIG. 2: Selective modulation of THz radiation. a, $\mathbf{b}$ Schematic representation of the measurements. A half-wave plate rotates the polarisation of a $650 \mathrm{~nm}$ pump beam by $2 \theta$, where $\theta$ is the angle between the vertical and the waveplate axis. This rotated pump beam selectively excites nanotubes aligned with the pump polarisation. $\mathrm{THz}$ waves are only absorbed by those photoexcited nanotubes that are aligned with the $\mathrm{THz}$ polarisation. $\mathbf{a} \theta=0$, and so excited nanotubes are aligned with the $\mathrm{THz}$ wave, yielding maximum absorption. $\mathbf{b} \theta=45^{\circ}$, so excited nanotubes are perpendicular to the $\mathrm{THz}$ polarisation, and so there is minimum absorption of the $\mathrm{THz}$ wave. c Photoinduced change in THz transmission, $\Delta T / T_{\mathrm{OFF}}$, equivalent to modulation depth of the device, as a function of halfwave plate angle $\theta$. The induced $\mathrm{THz}$ transmission change follows a cosine-squared relationship with waveplate angle, as expected from Malus' law, represented by the solid line.

As such, $\Delta T / T_{\mathrm{OFF}}$ is equivalent to the modulation depth of the nanotube $\mathrm{THz}$ modulator.

As expected from Malus' law for transmission through a polariser, the data display a cosine-squared relationship. At $0^{\circ}$, the pump pulse is polarised parallel to the $\mathrm{THz}$ probe, and nanotubes photoexcited by this pump pulse can absorb the $\mathrm{THz}$ probe, leading to maximum absorption. When the waveplate is rotated by $45^{\circ}$, the pump pulse is polarised perpendicular to the $\mathrm{THz}$ probe pulse, and so nanotubes photoexcited in this case absorb 

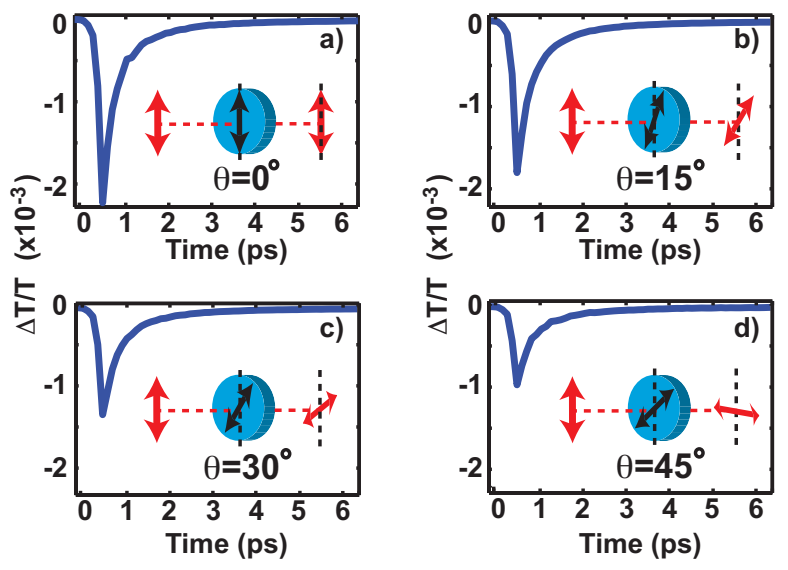

FIG. 3: Time dynamics of $\Delta T / T_{\mathrm{OFF}}$ as a function of time between optical pump and $\mathrm{THz}$ probe pulses, at waveplate angles of a) $0^{\circ}$, b) $15^{\circ}$, c) $30^{\circ}$ d) $45^{\circ}$.

much less of the $\mathrm{THz}$ radiation.

To analyse the transient nature of this polarisation, the time between photoexcitation by the pump and probing by $\mathrm{THz}$ pulses can be varied. Figures 3a-d display the modulation depth of the nanotubes at the peak of the $\mathrm{THz}$ pulse as a function of time after photoexcitation, for waveplate angles of $\theta=0^{\circ}, 15^{\circ}, 30^{\circ}$ and $45^{\circ}$. As can be seen from these figures, upon photoexcitation the modulation depth increases sharply, with a rise time of $500 \mathrm{fs}$. This is followed by a fall in modulation depth characterised by two exponential timescales - a first, rapid decay $\sim 400$ ps, followed by the decay of a small residual signal over several picoseconds. Thus ultrafast transient modulation of $\mathrm{THz}$ transmission can be achieved with the carbon nanotube polariser, at speeds suitable for communication technologies.

These dynamics can be understood by the creation and decay of excitons in the nanotubes. As discussed previously [23], photoexcitation preferentially generates excitons in those nanotubes parallel to the polarisation of the pump beam. Internal transitions within these excitons are in the $\mathrm{THz}$ range, and thus $\mathrm{THz}$ radiation incident upon the photoexcited nanotubes can be absorbed. The change in THz absorption decreases on two distinct timescales, corresponding to the decay of the generated excitons. At the high fluences used in these experiments, multiple excitons per nanotube are likely to be formed initially, leading to many-body interactions. These rapidly decay by Auger processes [22, 28, 29], leading to the first fast decay of photoinduced $\mathrm{THz}$ absorption. The longer timescale is then associated with single excitons. 
Our study demonstrates that ultrafast, dynamic control over the polarisation of $\mathrm{THz}$ radiation is possible with unaligned carbon nanotube films. With a modulation depth (related to $\Delta T / T)$ of $27 \mathrm{~dB}$, this technique has clear scope for improvement, e.g. through an increase in the density of nanotubes present in the sample, or alternatively by stacking of layers of the CNT samples yielding a thicker device. These techniques will be explored and developed in future work.

In summary, we have demonstrated the potential of unaligned, semiconducting carbon nanotubes for use in ultrafast $\mathrm{THz}$ modulators with sub-picosecond switch-on speeds. Photoexcitation by a polarised pump beam creates a tunable and transient polarisation of $\mathrm{THz}$ radiation through the nanotube device, which decays within a picosecond of the photoexcitation allowing for ultrafast modulation switching speeds. These properties, combined with the ease and cost effectiveness of production of the nanotube device, suggest promise for SWNT devices in $\mathrm{THz}$ communication technologies.

\section{Acknowledgements}

The authors would like to thank the EPSRC (UK) for financial support.

[1] R. Ulbricht, E. Hendry, J. Shan, T. F. Heinz, and M. Bonn, Rev. Mod. Phys. 83, 543 (2011).

[2] M. Tonouchi, Nat. Photon. 1, 97 (2007).

[3] B. S. Williams, Nat. Photonics 1, 517 (2007).

[4] J. Federici and L. Moeller, J. Appl. Phys. 107, 111101 (2010).

[5] F. Yan, C. Yu, H. Park, E. P. J. Parrott, and E. Pickwell-MacPherson, J. Infrared Millim. Terahertz Waves 34, 489 (2013).

[6] M. Rahm, J. S. Li, and W. J. Padilla, J. Infrared Millim. Terahertz Waves 34, 1 (2013).

[7] H. Alius and G. Dodel, 32, 1 (1991).

[8] T. Nozokido, H. Minamide, and K. Mizuno, Electron. Commun. Jpn. Pt. II-Electron. 80, 1 (1997).

[9] L. Fekete, F. Kadlec, P. Kužel, and H. Němec, Opt. Lett. 32, 680 (2007). 
[10] H. T. Chen, W. J. Padilla, J. M. O. Zide, A. C. Gossard, A. J. Taylor, and R. D. Averitt, Nature 444, 597 (2006).

[11] R. S. Yan, B. Sensale-rodriguez, L. Liu, D. Jena, and H. G. Xing, Opt. Express 20, 28664 (2012).

[12] C. F. Hsieh, Y. C. Lai, R. P. Pan, and C.-L. Pan, Opt. Lett. 33, 1174 (2008).

[13] H. Park, E. P. J. Parrott, F. Fan, M. Lim, H. Han, V. G. Chigrinov, and E. PickwellMacPherson, Opt. Express 20, 11899 (2012).

[14] Z. Huang, H. Park, E. P. J. Parrott, H. P. Chan, and E. Pickwell-MacPherson, IEEE Photonics Technol. Lett. 25, 81 (2013).

[15] H. Park, E. P. J. Parrott, Z. Huang, H. P. Chan, and E. Pickwell-MacPherson, Appl. Phys. Lett. 101, 121108 (2012).

[16] S. Shoji, H. Suzuki, R. P. Zaccaria, Z. Sekkat, and S. Kawata, Phys. Rev. B 77, 153407 (2008).

[17] B. G. Kang, Y. J. Lim, K. U. Jeong, K. Lee, Y. H. Lee, and S. H. Lee, Nanotechnology 21, $405202(2010)$.

[18] L. Ren, C. L. Pint, L. G. Booshenri, W. D. Rice, X. F. Wang, D. J. Hilton, K. Takeya, I. Kawayama, M. Tonouchi, R. H. Hauge, et al., Nano Lett. 9, 2610 (2009).

[19] L. Ren, C. L. Pint, T. Arikawa, K. Takeya, I. Kawayama, M. Tonouchi, R. H. Hauge, and J. Kono, Nano Lett. 12, 787 (2012).

[20] X. L. Xu, P. Parkinson, K.-C. Chuang, M. B. Johnston, R. J. Nicholas, and L. M. Herz, Phys. Rev. B 82, 085441 (2010).

[21] A. Hagen, M. Steiner, M. B. Raschke, C. Lienau, T. Hertel, H. H. Qian, A. J. Meixner, and A. Hartschuh, Phys. Rev. Lett. 95, 197401 (2005).

[22] L. B. Huang and T. D. Krauss, Phys. Rev. Lett. 96, 057407 (2006).

[23] X. Xu, K. Chuang, R. J. Nicholas, M. B. Johnston, and L. M. Herz, J. Phys. Chem. C 113, 18106 (2009).

[24] A. Nish, J. Y. Hwang, J. Doig, and R. J. Nicholas, Nat. Nanotechnol. 2, 640 (2007).

[25] S. D. Stranks, A. M. R. Baker, J. A. Alexander-Webber, B. Dirks, and R. J. Nicholas, Small 9, 2245 (2013).

[26] S. D. Stranks, S. N. Habisreutinger, B. Dirks, and R. J. Nicholas, Adv. Mater. 25, 4365 (2013).

[27] C. J. Docherty, C. Lin, H. J. Joyce, R. J. Nicholas, L. M. Herz, L. Li, and M. B. Johnston, Nat. Commun. 3, 1228 (2012). 
[28] F. Wang, G. Dukovic, L. E. Brus, and T. F. Heinz, Phys. Rev. Lett. 92, 177401 (2004).

[29] Y. Z. Ma, L. Valkunas, S. L. Dexheimer, S. M. Bachilo, and G. R. Fleming, Phys. Rev. Lett. 94, 157402 (2005). 